\title{
Positive outcomes enhance incidental learning for both younger and older adults
}

\section{Mara Mather* and Andrej Schoeke}

University of Southern California, Los Angeles, CA, USA

Edited by:

Gregory R. Samanez-Larkin,

Vanderbilt University, USA

\section{Reviewed by:}

Alan Castel, University of California Los Angeles, USA

Stefanie Brassen, University Medical

Center Hamburg-Eppendorf, Germany

\section{*Correspondence:}

Mara Mather, 3715 McClintock Ave., University of Southern California Los Angeles, Los Angeles, CA 90089, USA.

e-mail:mara.mather@usc.edu
Previous studies suggest that memory encoding is enhanced when people are anticipating a potential reward, consistent with the idea that dopaminergic systems that respond to motivationally relevant information also enhance memory for that information. In the current study, we examined how anticipating and receiving rewards versus losses affect incidental learning of information. In addition, we compared the modulatory effects of reward anticipation and outcome on memory for younger and older adults. Forty-two younger (aged 18-33 years) and 44 older (aged 66-92 years) adults played a game involving pressing a button as soon as they saw a target. Gain trials began with a cue that they would win $\$ 0.25$ if they pressed the button fast enough, loss trials began with a cue that they would avoid losing $\$ 0.25$ if they pressed the button fast enough, and no-outcome trials began with a cue indicating no monetary outcome. The target was a different photoobject on each trial (e.g., balloon, dolphin) and performance outcomes were displayed after the photo disappeared. Both younger and older adults recalled and recognized pictures from trials with positive outcomes (either rewarding or loss avoiding) better than from trials with negative outcomes. Positive outcomes were associated with not only enhanced memory for the picture just seen in that trial, but also with enhanced memory for the pictures shown in the next two trials. Although anticipating a reward also enhanced incidental memory, this effect was seen only in recognition memory of positive pictures and was a smaller effect than the outcome effect. The fact that older adults showed similar incidental memory effects of reward anticipation and outcome as younger adults suggests that reward-memory system interactions remain intact in older age.

Keywords: aging, reward outcome, incidental memory and learning, monetary incentive delay task, valence, picture recognition

\section{INTRODUCTION}

Most of what we experience everyday is quickly forgotten, if it is even encoded in the first place. Yet the human brain is remarkably effective at learning about things that matter. Our memory systems rely on a variety of signals to distinguish things that matter from things that do not, such as the probability of encountering information again given the pattern of previous exposure to that information (Anderson and Schooler, 2000; Kornell et al., 2010) or levels of arousal during learning (Mather and Sutherland, 2011). Recent work has started to examine whether receiving or anticipating a reward is another factor that modulates memory encoding and consolidation processes. Prioritizing memory encoding for information learned around the time of receiving a reward could have utility. For instance, it may be useful to remember what one did or saw just before obtaining a positive outcome in order to replicate the outcome in the future.

Several recent studies with humans suggest that anticipating rewards can enhance memory. For instance, participants who studied lists of items with some items promising high rewards if remembered later had better long-term memory for the highreward items (Adcock et al., 2006; Callan and Schweighofer, 2008). Greater activity in the midbrain, nucleus accumbens, and hippocampus during study predicted better memory performance later (Adcock et al., 2006). Such findings suggest that activating neural pathways involved in reward processing enhances memory - but it is also possible that this pattern of brain activity was not the critical factor enhancing memory - the enhanced memory may have resulted from the more effortful encoding for the items that would get a larger reward when remembered later, at the same time that reward regions activated at the prospect of a potential future reward.

However, other studies suggest that enhanced memory for information learned during reward anticipation can occur even when memory for the information itself is not tied with the future reward. For instance, a couple of studies showed objects as cues; whether the object was living or non-living was the signal indicating whether participants could expect a reward if they executed the upcoming task (indicating whether a target number was larger or smaller than five) fast enough (Wittmann et al., 2005; Bialleck et al., 2011). Although the object category was relevant to the reward, the specifics of the objects were irrelevant. In both studies, participants remembered the objects that had predicted reward better than those that had not. Activity in the midbrain (specifically, the substantia nigra) and hippocampus during the 
initial viewing of the rewarding object cues predicted subsequent memory for them, but midbrain activity did not predict memory for neutral object cues (Wittmann et al., 2005).

In another study using the same number comparison task (Wittmann et al., 2008a), memory was tested for pictures whose content was entirely irrelevant to the anticipated rewards. Positive, neutral, or negative pictures were placed behind a fixation point that was green to signal potential reward and yellow to signal no potential reward. Participants remembered positive pictures seen behind the green cues better than positive pictures seen behind the yellow cues, but reward anticipation did not affect incidental memory for negative or neutral pictures. Thus, positive emotional valence seems to interact with the reward system to enhance memory formation further.

One limitation of the previous studies showing enhanced incidental memory on rewarding trials (Wittmann et al., 2005, 2008b; Bialleck et al., 2011) is that they did not separate the effects of reward anticipation and reward delivery. In those studies, it was not clear whether memory enhancements were due to anticipating rewards or to retroactive enhancement of the initial cues once the reward was received later in the trial.

Another limitation of the studies described above is that they were all conducted with younger adults, leaving open the question of whether older adults show similar or different influences of reward processing on incidental memory encoding. Aging is associated with changes in neural systems and brain regions linked with reward processing (Marschner et al., 2005; Backman et al., 2010) as well as with changes in memory processes (Hedden and Gabrieli, 2004; Luo and Craik, 2008; Mather, 2010). Thus, one cannot assume that reward anticipation or delivery will affect memory encoding in the same way for older adults as for younger adults.

Although the question of whether there are age differences in how the process of anticipating or receiving a reward influences memory encoding of novel information has not been tackled directly in the literature, there are some related findings. For instance, previous studies have examined whether there are age differences in the ability to learn stimulus-reward contingencies. Such studies reveal that older adults take longer than younger adults to learn which letters or pictures are probabilistically associated with higher point outcomes (Mell et al., 2005, 2009; Eppinger et al., 2010; Eppinger and Kray, 2011). However, an important point to note is that findings that older adults are worse at learning associations between reward and certain cues may be driven by age-related impairments in associative memory (e.g., Mitchell et al., 2000; Naveh-Benjamin et al., 2004) rather than by age-related changes in how reward processing modulates memory.

Indeed, there is some evidence that argues against the hypothesis that older adults show poorer stimulus-outcome learning because of decline in reward processing. One piece of evidence is that when reward modulation and stimulus-reward associative learning are measured separately, reward modulation of item learning is as strong among older adults as among younger adults, even when older adults show impaired stimulus-reward associative learning (Eppinger et al., 2010). In Eppinger et al.'s study, participants made two-choice decisions between two pictures of objects. Some of the objects were seen repeatedly in a positive learning task, in which feedback was either a gain of 50 cents or a gain of 0 cents. Other objects were seen repeatedly in a negative learning task, in which feedback was either a loss of 50 cents or a loss of 0 cents. Each object always was always associated with the same outcome (a gain, no monetary outcome, or a loss). After completing the learning task, participants were shown the objects from the positive and negative learning tasks intermixed with new objects and asked to identify which objects they had seen before. Although older adults showed impaired stimulus-outcome learning during the learning tasks (they were less likely to choose the objects that predicted better outcomes), both younger and older adults had better item recognition memory for the objects seen in the positive learning condition than those seen in the negative learning condition, and there was no age difference in the size of this advantage.

This finding that the type of reward outcome modulates memory similarly in younger and older adults is consistent with another study in which participants had to try to select the correct symbol-color association on each trial (Weiler et al., 2008). Participants slowly learned the correct associations across the trials and performance was better for the two symbols for which correct responses were rewarded with 20 cents than for the two symbols for which correct responses were rewarded with 5 cents. This rewardenhancement in symbol-color associative learning was similar in magnitude for younger and older adults.

Another relevant pattern from previous research is that older adults effectively prioritize their explicit memory to focus on high value information (Castel, 2008). For instance, when presented briefly with one word at a time together with the point value that remembering that word would yield, older adults were as likely as younger adults to recall the highest point value words despite having overall lower recall (Castel et al., 2002).

In summary, previous research with younger adults indicates that anticipating or receiving rewards can enhance concurrent memory encoding (Wittmann et al., 2005, 2008b; Bialleck et al., 2011), but these studies did not distinguish clearly between the effects of reward anticipation and delivery. In addition, two studies (Weiler et al., 2008; Eppinger et al., 2010) provide initial evidence that rewarding outcomes modulate memory for information seen just beforehand to a similar extent in younger and older adults. However, in both studies, the stimuli were each presented many times and learning the information helped to obtain the rewarding outcomes. In the current study, we were interested in whether rewarding outcomes modulate incidental memory encoding of novel information presented just once. In addition, we examined the independent contributions of reward anticipation and outcome.

To investigate these questions, we modified the Monetary Incentive Delay task (Knutson et al., 2001). In our version of the task (Figure 1), participants saw a cue on each trial that indicated whether they could earn money, avoid losing money, or have no monetary outcome from responding to the upcoming target quickly enough. The target was a novel object on each trial, and after they pressed the key in response to the target, participants received feedback about the outcome. After completing this response time task, participants then completed a surprise recognition memory test for the objects. We examined whether the anticipation or outcome type on each trial affected incidental 


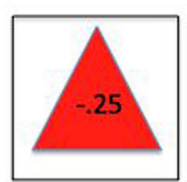

$2 \mathrm{~s}$

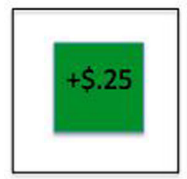

$2 \mathrm{~s}$

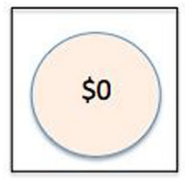

$2 \mathrm{~s}$

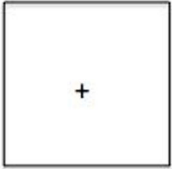

$2-4 s$

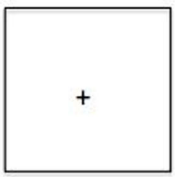

$2-4 s$

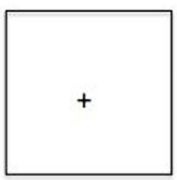

$2-4 s$

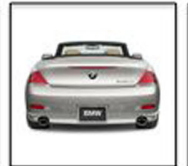

$2 \mathrm{~s}$

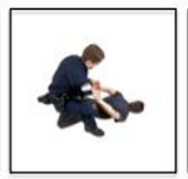

$2 \mathrm{~s}$

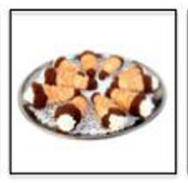

$2 \mathrm{~s}$

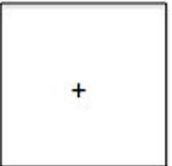

$2 \mathrm{~s}$

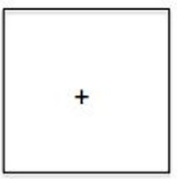

$2 \mathrm{~s}$

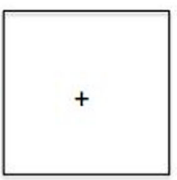

$2 \mathrm{~s}$

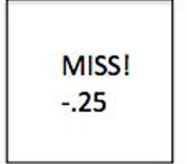

$2 \mathrm{~s}$

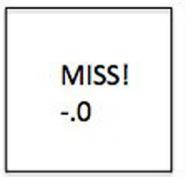

$2 \mathrm{~s}$

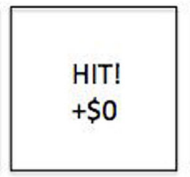

$2 \mathrm{~s}$

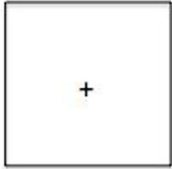

$2 \mathrm{~s}$

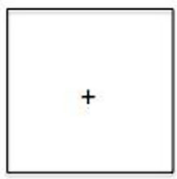

$2 \mathrm{~s}$

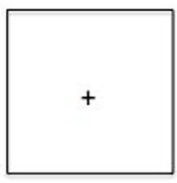

$2 \mathrm{~s}$

FIGURE 1 | Examples of experiment trials, with a loss-anticipation trial in the first row, gain anticipation in the second row and no anticipated monetary outcome in the third row. Participants were asked to hit a key as quickly as they could when they saw a picture, then they received feedback about whether they responded quickly enough.

memory for the target object and whether such effects differed for younger versus older adults. In addition, because previous research suggests that reward anticipation has a larger benefit for memory encoding when the to-be-remembered information is emotionally positive than when it is negative (Wittmann et al., 2008a), we compared the effects of reward anticipation and outcome on memory for emotionally positive versus negative stimuli.

\section{MATERIALS AND METHODS PARTICIPANTS}

Forty-two younger adults (aged 18-33 years; $M=21.6, \mathrm{SD}=3.4$, 30 females and 12 males) and 44 older adults (aged 66-92 years; $M=74.5, \mathrm{SD}=5.7,31$ females and 13 males) completed the study. Participants were recruited through a list of research volunteers that was obtained via newspaper and online ads, fliers at senior centers and public places, and letters to University of Southern California (USC) alumni. Some younger adult participants were recruited through the USC Psychology participant pool. Participants received monetary compensation or course credit for their time as well as earnings received during the task. Younger adults had completed fewer years of education $(M=14.6, \mathrm{SD}=1.62)$ than the older adults $(M=17.34, \mathrm{SD}=3.12), t(84)=4.99$, $p<0.001$, and also scored lower on the Nelson-Denny vocabulary test (Brown et al., 1993; $M=16.0, \mathrm{SD}=3.3$ ) than the older adults $(M=20.1, \mathrm{SD}=2.6), t(84)=6.3, p<0.001$. Scores on the 20 item Center for Epidemiological Studies Depression Scale (CESD; Radloff, 1977) indicated slightly more symptoms of depression among younger adults $(M=14.4, \mathrm{SD}=9.2)$ than among older adults $(M=10.6, \mathrm{SD}=8.5), t(84)=2.01, p=0.048$. Mood scores on the Positive and Negative Affect Scale (PANAS; Watson et al., 1988) did not differ significantly for negative affect ( $M$ younger $=13.1, \mathrm{SD}=3.5 ; M$ older $=12.4, \mathrm{SD}=3.6$ ), but positive affect was lower among younger adults $(M=29.5, \mathrm{SD}=7.2)$ than among older adults $(M=33.8, \mathrm{SD}=6.8), t(84)=2.83, p=0.01$. On a scale of 1 being "very poor health" and 9 being "excellent health," younger $(M=7.5, \mathrm{SD}=0.9)$ and older adults $(M=7.6$, $\mathrm{SD}=0.9$ ) did not differ significantly. Self ratings of how "your stress level is today on a scale of $1-9$ " with 1 being very low and 9 very high also did not differ significantly by age group ( $M$ younger $=4.3, \mathrm{SD}=2.0 ; M$ older $=4.2, \mathrm{SD}=2.4$ ).

\section{MATERIALS}

Ninety-six photo-objects were used for target items and 40 photo-objects as filler new items on the recognition test, selected from photo-objects used in previous studies with older adults (Kensinger et al., 2007). Half of the items within each set were mildly positive (e.g., sports car, dessert) and half were mildly negative (police officer handcuffing someone, knife).

\section{PROCEDURE}

After giving informed consent, participants completed a practice section in which the response deadline for the task was calibrated so that they could respond quickly enough about two-third of the time. Next, in the actual experiment task, participants played 96 trials in a response speed game. Each trial involved a cue to let participants know whether responding fast enough to a target picture would lead to a gain, loss, or no monetary outcome (Figure 1). After the 2-s presentation of the cue, they saw a fixation cross for $2 \mathrm{~s}$ plus a random number between 0 and $2.5 \mathrm{~s}$ (for a total of $2-4.5 \mathrm{~s}$ ) and then the target picture of a positive or negative photo-object for $2 \mathrm{~s}$. Their task was to respond to the picture as quickly as they could before a beep indicating the response deadline (which was dynamically calibrated throughout the task based on performance on that type of trial). Regardless of the response 
deadline, the picture remained visible for the full $2 \mathrm{~s}$. After the picture disappeared, they were shown a fixation cross for $2 \mathrm{~s}$, then feedback about their performance and any gain or loss (e.g." "Miss! $-\$ 0.25$ ”) for $2 \mathrm{~s}$. The trial ended with a fixation cross for $2 \mathrm{~s}$.

After the response speed game, participants completed vocabulary, health, and other questionnaires for $10 \mathrm{~min}$. They then were given a pen and paper and $5 \mathrm{~min}$ to recall and list descriptions of as many of the target pictures as they could. Next, in a recognition memory test, they were shown each of the photo-objects from the game as well as new photo-objects, in a random order and asked to indicate whether they saw each one during the response speed game or not with a simple yes/no judgment. Finally, they rated each picture for valence on a $1-9$ scale $(1=$ very negative, $9=$ very positive) and for arousal on a $1-9$ scale $(1=$ not at all arousing or intense, $9=$ very arousing or intense). At the end of the session, participants were paid their winnings in addition to their regular compensation or credit for the study session. Winnings ranged from $\$ 5.25$ to $\$ 8.75(M=\$ 7.44, \mathrm{SD}=0.80)$ and there was no significant difference in amounts for younger and older adults.

\section{RECALL CODING}

Two coders labeled each picture description to indicate which picture in the stimuli set it corresponded with, or whether it did not match any of the pictures seen. There was $89 \%$ agreement between the two coders on the exact pictures the descriptions matched. If the two coders disagreed, one of the coders reviewed the item and made a final judgment. The criterion for successful recall of a picture was that coders could identify a specific image that fit the participant's description. After coding was complete, for each participant, we linked each recalled item to the parameters of the original trial on which it was seen (i.e., was it a reward, loss, or no anticipation trial; did it have a hit or miss outcome).

\section{RESULTS}

Initial analyses revealed that one younger female failed to follow instructions (she called every new item old during the recognition test). Her data were excluded from the analyses below.

\section{RESPONSE TIMES}

There were two issues of interest for the response times. The first was whether our adaptive algorithm had equalized the proportion of responses during the MID task that were counted as having been made within the response deadline for each type of trial type. In other words, were younger and older participants equally likely to get positive feedback and was positive-feedback equally likely for each trial type? We confirmed that positive outcomes were equally distributed by analyzing the proportion of responses that yielded a "hit" feedback in a 3 (anticipation: loss, none, gain) $\times 2$ (item valence: negative, positive) $\times 2$ (age group: younger, older) ANOVA. As intended, on average, $67 \%$ of responses were made within each respective deadline and there were no significant main effects or interactions, indicating that about the same number of responses were counted as hits for each type of trial for younger and older adults.

The second question for the response times was whether they varied depending on what type of outcome was anticipated. Repeating the above ANOVA with mean MID task response time as the dependent measure revealed a significant effect of age group, $F(1,83)=23.94$, MSE $=680782, p<0.001, \eta_{p}^{2}=0.22$, as younger adults responded faster $(M=266 \mathrm{~ms}, \mathrm{SE}=17)$ than older adults $(M=380 \mathrm{~ms}, \mathrm{SE}=16)$. In addition, there was a significant interaction of anticipation type and age group, $F(2,166)=4.58$, $\mathrm{MSE}=3731, p=0.01, \eta_{p}^{2}=0.05$, as younger participants responded slower when there was no potential monetary outcome $(M=286 \mathrm{~ms}, \mathrm{SE}=17)$ than when there was a potential for loss $(M=254 \mathrm{~ms}, \mathrm{SE}=19)$ or a potential for gain $(M=259 \mathrm{~ms}$, $\mathrm{SE}=17)$, whereas older adults showed little difference between the no-outcome condition $(M=376 \mathrm{~ms}, \mathrm{SE}=16)$ and the loss $(M=380 \mathrm{~ms}, \mathrm{SE}=18)$ or gain conditions $(M=383 \mathrm{~ms}, \mathrm{SE}=16)$. There were no other significant effects.

\section{RECALL}

On average, participants recalled about 10 pictures, with no significant difference between age groups (younger $M=10.8, \mathrm{SE}=0.9$; older $M=10.0, \mathrm{SE}=0.9$ ). We examined how the context in which a particular picture was seen during the MID task affected later memory for it. The two context variables were anticipation type and response outcome from the trial in which that picture was originally seen. A 3 (anticipation: loss, none, gain) $\times 2$ (item valence: negative, positive) $\times 2$ (MID task outcome: hit, miss) $\times 2$ (age group: younger, older) ANOVA with the proportion of previously seen items that were recalled from each category revealed that participants were more likely to recall items from the MID task hit trials $(M=0.12, \mathrm{SE}=0.01)$ than from the response time miss trials $(M=0.09, \mathrm{SE}=0.01), F(1,83)=11.21, \mathrm{MSE}=0.01$, $p=0.001, \eta_{p}^{2}=0.12^{1}$. This enhanced recall for target items from hit trials was seen for both younger and older adults (Figure 2). Response outcome also showed a marginally significant interaction with anticipation type, such that the memorial benefit of a rewarding outcome was seen more on trials with anticipated monetary outcomes than on trials with no anticipated monetary outcome (effect shown separately for younger and older adults in Figure 3), $F(2,166)=2.91, \mathrm{MSE}=0.01, p=0.06, \eta_{p}^{2}=0.03$. There were no other significant effects.

\section{RECOGNITION MEMORY}

Because our hypotheses focused on comparing how different types of encoding contexts affect later memory for the pictures, we

\footnotetext{
${ }^{1}$ To examine whether the feedback outcome effect could be accounted for by response time differences for trials receiving positive feedback versus negative feedback, we compared the effect of outcome on both slow and fast response times. For each participant, we computed the median response time separately for each type of anticipation (reward, none, loss). We then categorized each old item as having had a response during the MID task that was above or below the median response time in its anticipation type category and computed the proportion of slow and fast-response items were recalled. A 2 (response time: slow, fast) $\times 2$ (MID task outcome: hit, miss) $\times 2$ (age group: younger, older) ANOVA confirmed the main effect of task outcome seen in the other analysis, $F(1,83)=6.64, \mathrm{MSE}=0.005$, $p=0.01, \eta_{p}^{2}=0.07$ and revealed no interaction of task outcome and response time $(p>0.9)$ and no other significant effects. Post hoc $t$-tests showed that the enhancement for items followed by positive outcomes was independently significant for items from fast response trials $(M$ hit $=0.12, \mathrm{SE}=0.01 ; M$ miss $=0.10, \mathrm{SE}=0.01$ ), $t(84)=2.18, p=0.03$, and marginally significant for items from slow response trials $(M$ hit $=0.11, \mathrm{SE}=0.01 ; M$ miss $=0.09, \mathrm{SE}=0.01), t(84)=1.83, p=0.07$. Thus, the effect of feedback was similar across performance levels.
} 
Initial Viewing of Item was Followed by Positive Feedback

InitialViewing of Item was Followed by Negative Feedback

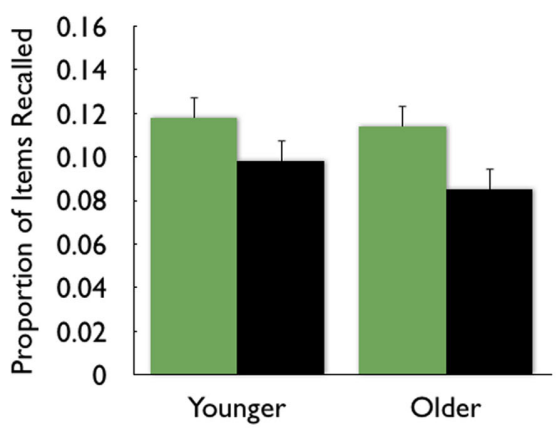

FIGURE 2 | Both younger and older adults were more likely to recall pictures that were followed by positive feedback than those followed by negative feedback.

Initial Viewing of Item was Followed by Positive Feedback

Initial Viewing of Item was Followed by Negative Feedback

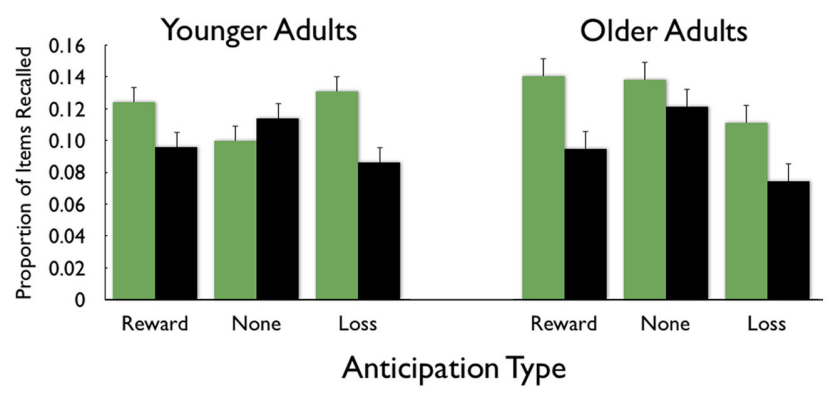

FIGURE 3 | Greater recall of pictures followed by positive feedback was seen for feedback associated with monetary outcomes more than for feedback with no monetary outcome.

could only include old pictures in our primary analyses (new pictures could not be categorized by encoding context). However, to get an indication of overall memory accuracy, we used a 2 (item valence: negative, positive) $\times 2$ (age group: younger, older) ANOVA with $d^{\prime}$ recognition accuracy measures as the dependent variable. There was a significant effect of valence, $F(1,83)=6.58$, $\mathrm{MSE}=0.27, p<0.001, \eta_{p}^{2}=0.27$, with $d^{\prime}$ higher for positive $(M=2.08, \mathrm{SE}=0.08)$ than for negative $(M=1.69, \mathrm{SE}=0.08)$ pictures. Neither the main effect of age group nor the interaction was significant (both $F<1$ ). In contrast with the lack of age differences in accuracy, repeating the ANOVA using the response bias measure $C$ (Macmillan and Creelman, 1991) as the dependent variable revealed that older adults were significantly more biased to call pictures old $(M=0.39, \mathrm{SE}=0.06)$ than younger adults were $(M=0.65, \mathrm{SE}=0.06), F(1,83)=10.31, \mathrm{MSE}=0.28$, $p=0.002, \eta_{p}^{2}=0.11$. Overall, participants were more likely to call positive pictures old $(M=0.39, \mathrm{SE}=0.04)$ than to call negative pictures old $(M=0.66, \mathrm{SE}=0.05), F(1,83)=46.30, \mathrm{MSE}=0.07$, $p<0.001, \eta_{p}^{2}=0.36$, and there was not a significant interaction of age and valence $(F<1)^{2}$.

Next, we turned to our main focus comparing the effects of reward anticipation and outcome on incidental memory. We conducted a 3 (anticipation: loss, none, gain) $\times 2$ (item valence: negative, positive $) \times 2$ (MID task outcome: hit, miss $) \times 2$ (age group: younger, older) ANOVA with the proportion of old target pictures correctly identified as old as the dependent measure. Consistent with the age difference in response bias reported above, older adults were more likely to identify the pictures as old $(M=0.68$, $\mathrm{SE}=0.02)$ than the younger adults were $(M=0.60, \mathrm{SE}=0.02)$, $F(1,83)=8.24, \mathrm{MSE}=0.22, p=0.005, \eta_{p}^{2}=0.09$. As in the recall data, there was a large effect of MID task outcome, $F(1,83)=24.99$, $\mathrm{MSE}=0.05, p<0.001, \eta_{p}^{2}=0.23$, with participants recognizing pictures from trials in which they got "hit" feedback $(M=0.67$, $\mathrm{SE}=0.02$ ) better than pictures from trials in which they got "miss" feedback $(M=0.60, \mathrm{SE}=0.02$; see Figure 4 for effect separately for younger and older adults $)^{3}$.

There also was an interaction of item valence and anticipation, $F(2,166)=4.00$, MSE $=0.03, p=0.02, \eta_{p}^{2}=0.05$. Participants remembered positive items best in the reward-anticipation condition $(M=0.65, \mathrm{SE}=0.02)$, followed by the no-outcome condition $(M=0.63, \mathrm{SE}=0.02)$ then by the loss-anticipation condition $(M=0.59, \mathrm{SE}=0.02)$. For the negative items, anticipation type had less effect $\left(M_{\text {reward }}=0.64, \mathrm{SE}=0.02, M_{\text {none }}=0.66\right.$, $\left.\mathrm{SE}=0.02, M_{\text {loss }}=0.65, \mathrm{SE}=0.02\right)$.

Thus, both anticipation of whether there was a potential reward or loss and actually getting positive or negative-feedback influenced incidental memory for the response time target items, although the anticipation effect was modulated by whether the items were negative or positive. The feedback effect was particularly strong.

\section{LINGERING EFFECTS OF POSITIVE OUTCOMES}

The analyses above revealed that getting positive feedback enhanced later memory for the item from that trial. In additional exploratory analyses, we compared the proportion of recognized pictures that had been seen on trials with positive feedback on the

${ }^{2}$ In terms of the raw hits (proportion of old items called old), older adults had significantly more $(\mathrm{M}=0.69, \mathrm{SE}=0.02)$ than younger adults $(M=0.61, \mathrm{SE}=0.02)$, $F(1,83)=9.02, \mathrm{MSE}=0.04, p=0.004, \eta_{p}^{2}=0.10$. Consistent with their more lenient criterion to call pictures old, older adults also had more false alarms $(M=0.13, \mathrm{SE}=0.02)$ than younger adults $(M=0.09, \mathrm{SE}=0.02), F(1,83)=3.68$, $\mathrm{MSE}=0.02, p=0.058, \eta_{p}^{2}=0.04$.

${ }^{3}$ As done with the recalled items (see footnote 1 ), we categorized each old item on the recognition test by whether it had been responded to faster or slower than the median response for items in the same type of trial (reward, none, or loss anticipation). A 2 (response time: slow, fast) $\times 2$ (MID task outcome: hit, miss) $\times 2$ (age group: younger, older) ANOVA revealed that, as in the previous analyses, participants were more likely to recognize items from trials with positive feedback $(M=0.67, \mathrm{SE}=0.02)$ than from trials with negative feedback $(M=0.61$, $\mathrm{SE}=0.02), F(1,84)=12.91, \mathrm{MSE}=0.02, p=0.001, \eta_{p}^{2}=0.13$. Other than a significant main effect of age as in the main analysis, there were no significant effects ( $p>0.6$ for the interaction of outcome and response time). In addition, $t$-tests revealed that the effects of feedback were significant for both the slow $(M$ hit $=0.66$, $\mathrm{SE}=0.02 ; M$ miss $=0.60, \mathrm{SE}=0.02), t(84)=3.38, p=0.001$, and the fast responses $(M$ hit $=0.68, \mathrm{SE}=0.02, M$ miss $=0.63, \mathrm{SE}=0.02), t(84)=2.19, p=0.03$. Thus, feedback outcomes influenced whether the target would later be remembered both when response time performance was good and when it was poor. 
Initial Viewing of Item was Followed by Positive Feedback Initial Viewing of Item was Followed by Negative Feedback

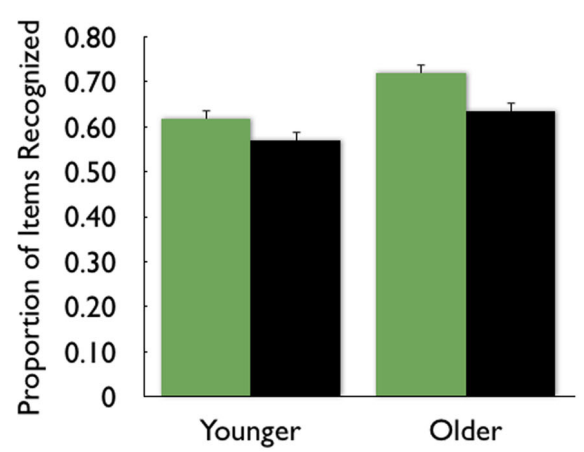

FIGURE 4 | Both younger and older adults were more likely to recognize pictures that were followed by positive feedback than those followed by negative feedback.

preceding trial versus the proportion of non-recognized pictures that had been seen on trials with positive feedback on the preceding trial. As shown in the middle two bars of Figure 5, recognized pictures were more likely to have been preceded by trials with positive feedback than were forgotten pictures. There was a similar effect for the $N-2$ trials, as well. These effects were significant in exploratory $t$-tests, whereas anticipation on one trial did not have lingering effects on subsequent trials ${ }^{4}$.

To follow up on these observations and examine whether the outcome on a preceding trial affected memory for the target in the current trial above and beyond the effects of outcomes from other preceding trials or from the current trial, we used hierarchical linear model (HLM) analyses. In these analyses, we examined whether getting positive feedback on one trial would have lingering enhancing effects on memory for items seen on subsequent trials. For each old item we categorized: (1) whether it was recognized or forgotten on the recognition memory test; (2) feedback outcome on the current trial; (3) feedback outcome on the previous trial; and (4) feedback outcome on the trial two back from the current trial. As all values were 1 or 0 , we specified Bernoulli model distributions for the two-level HLM analyses. The outcome variable was the recognition (or recall) outcome for each item, age group was a level-1 predictor, and feedback outcomes (items 2-4 above) were level-2 predictor variables. We included interactions of each level-2 predictor with age group in the model.

The analyses revealed that, even when controlling for the current trial outcome and the other preceding trial outcome, recognized items were more likely to have trials one and two back with positive feedback than were forgotten items (see Table 1 for statistics). Thus, positive feedback on one trial was associated with enhanced memory encoding for the unrelated target

${ }^{4}$ We also did not find any significant relationships between subsequent memory for the target on trial $N$ by reward anticipation type or feedback type on trial $N+1$ or $N+2$.

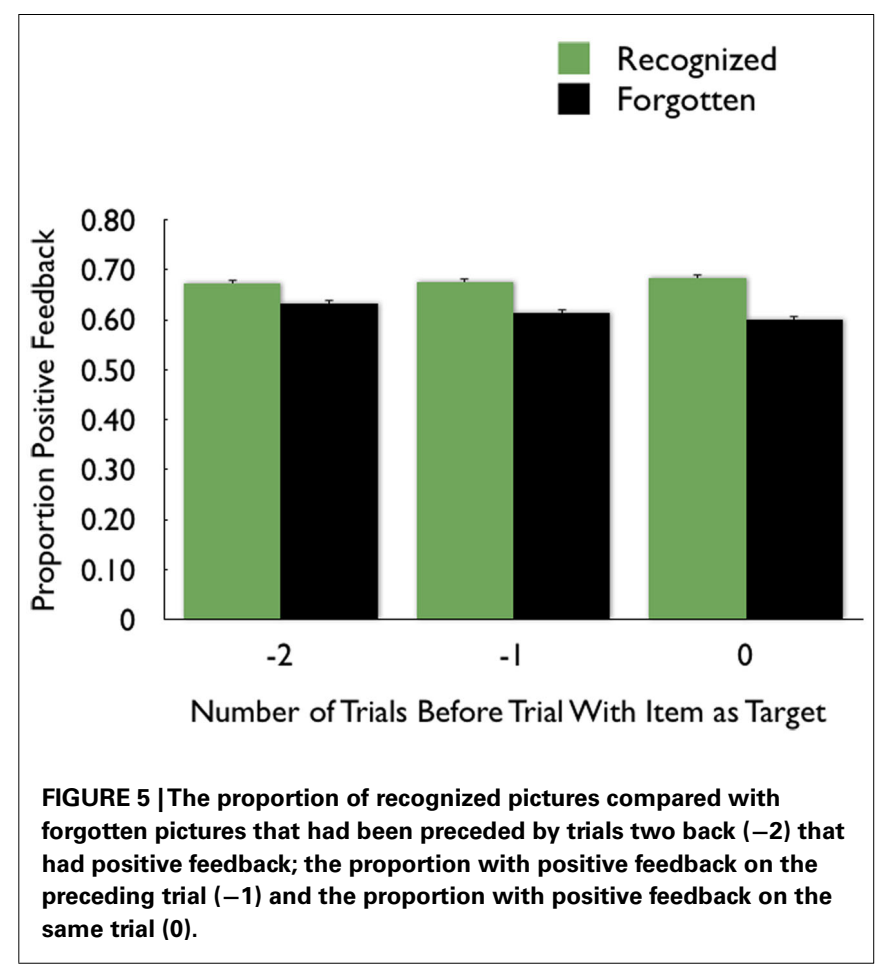

item on the next trial, and even on the next trial after that. While there was a significant age group effect on the intercept (consistent with the greater likelihood of calling pictures old seen among older adults), there were no other significant age group interactions.

We repeated the above analyses for recalled versus non-recalled items. Although the pattern was similar (Figure 6) to that seen in recognition memory, outcomes on the two preceding trials did not significantly affect picture recall (in part perhaps because small numbers of recalled items for some participants led to greater variability). However, as in the earlier analyses, there was a significant effect of the outcome on the current trial on later memory for that picture.

\section{PICTURE RATINGS ${ }^{5}$}

Positive pictures were given higher valence ratings $(M=6.49$, $\mathrm{SE}=0.09)$ than negative pictures $(M=3.39, \mathrm{SE}=0.09)$, $F(1,80)=433.31, \mathrm{MSE}=2.34, p<0.001, \eta_{p}^{2}=0.84$. There were no significant effects of age group on the valence ratings. For the arousal ratings, there was not a significant main effect of picture valence, but there was an interaction of age group by valence category, $F(1,81)=7.72, \mathrm{MSE}=4.31, p=0.007, \eta_{p}^{2}=$ 0.09 . Younger adults rated the negative pictures as more arousing $(M=4.06, \mathrm{SE}=0.25)$ than the positive pictures $(M=3.33$, $\mathrm{SE}=0.26)$, whereas the older adults rated the negative pictures as less arousing $(M=4.00, \mathrm{SE}=0.25)$ than the positive pictures $(M=4.31, \mathrm{SE}=0.26)$.

${ }^{5}$ Due to time constraints, three participants did not complete the post-experiment valence ratings of pictures. Of these, two did not complete the arousal ratings either. 
Table 1 | Hierarchical linear model (HLM) analysis beta coefficients ( $\beta$ ), robust standard errors (SE), $t$ ratios ( $t$ ), degrees of freedom (df), and $p$ values using the outcome on the current and preceding two trials to predict recognition (A) and recall (B) of the current trial's target picture.

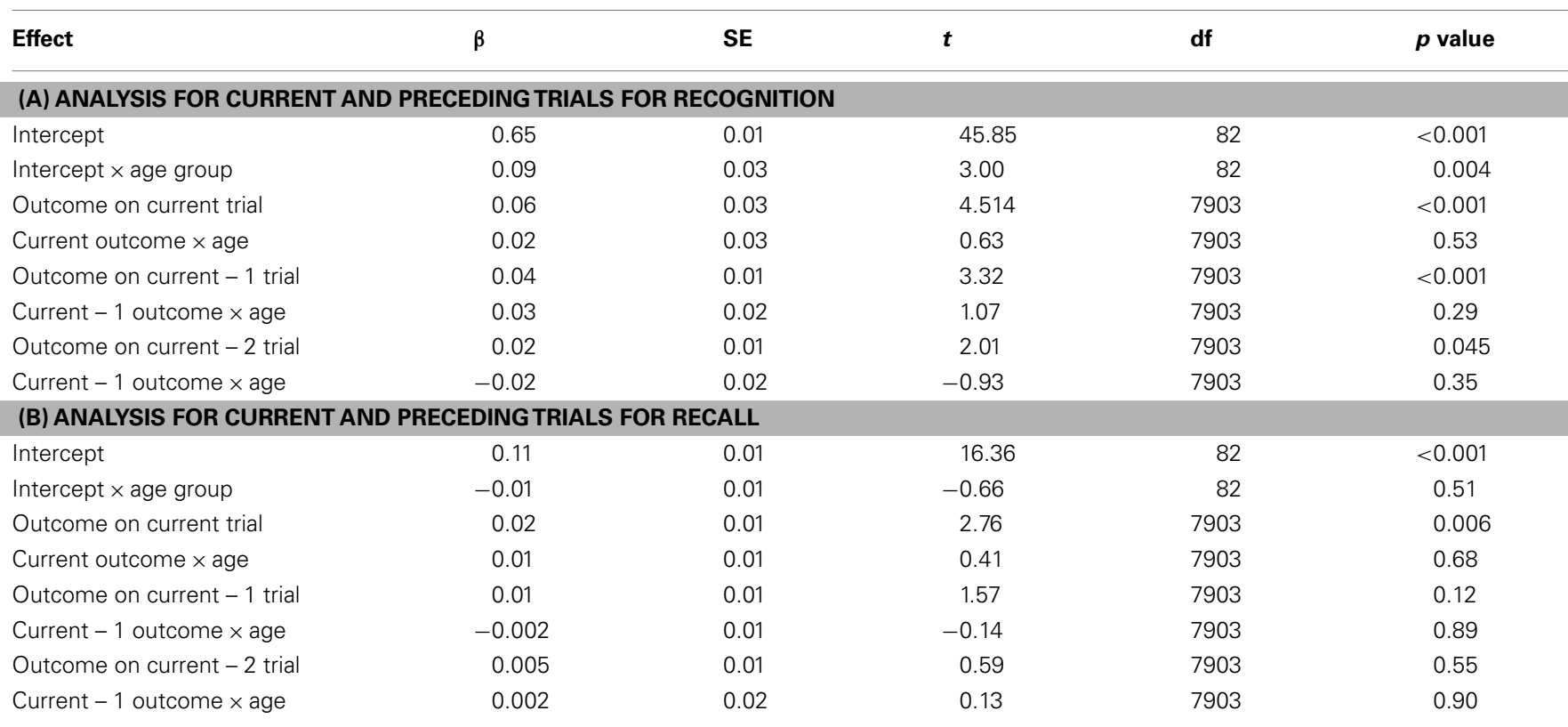

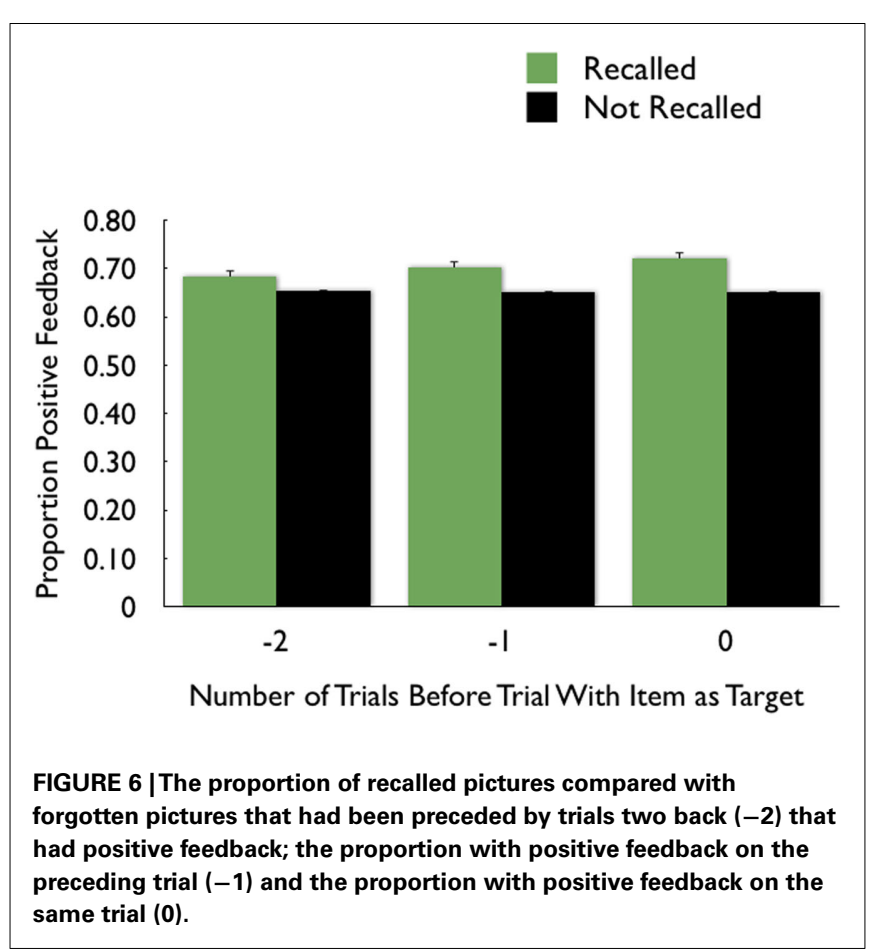

\section{DISCUSSION}

\section{EFFECTS OF ANTICIPATION AND OUTCOMES ON INCIDENTAL} LEARNING

In this study, we examined the effects of reward and loss anticipation as well as the effects of positive and negative outcome feedback (reaction time "hit" versus "miss" feedback) on incidental memory for items shown as response time targets (Figure 1). We found that the most robust effects were from whether the feedback was positive or negative. In both the reward and lossanticipation conditions, targets from positive-feedback trials were remembered better than targets from negative-feedback trials. This similar effect across reward and loss conditions was seen despite the fact that the positive feedback was associated with monetary reward in the reward condition and with a lack of a monetary loss in the loss condition. Thus, it seems that the outcome being positive relative to expectations is what matters, rather than the absolute amount of the reward. In addition, the enhancement in memory due to positive feedback was seen for both recall and recognition memory.

In recognition memory, anticipation type also influenced incidental memory, but only for the positively valenced items. The positive items were remembered best from reward-anticipation trials and worst from loss-anticipation trials. Thus, while anticipating a reward did enhance memory, its influence was not seen across all item types and did not show up in recall. In contrast, positive feedback was associated with better memory than negativefeedback nearly across the board, with the exception of recall of pictures from trials with no monetary outcomes (Figure 3). From these results, it seems that both anticipating and receiving positive outcomes modulates memory, but that reward delivery has a larger impact. Previous studies have not distinguished the effects of reward anticipation and delivery (Wittmann et al., 2005, 2008b) and so effects from those studies that have been interpreted as being due to reward anticipation may actually have been influenced by reward delivery.

In addition to having a strong effect on memory for items previously seen in that trial, positive feedback on one trial was associated with better later recognition of the targets in the subsequent trial and even in the trial after that (Figure 5). HLM analyses revealed that the significant relationship between the outcomes on the preceding trials and later memory for the target picture on the current 
trial held up even when the influence of the current trial outcome was factored out. These findings suggest that positive outcomes can enhance memory-encoding processes in the period after the positive event (in this case, for the next 20 s or so).

\section{EFFECTS OF ANTICIPATION AND OUTCOMES WERE SIMILAR FOR YOUNGER AND OLDER ADULTS}

As reviewed above, our study revealed three main findings about how the reward/loss context affected incidental encoding. First, reward anticipation did not have much effect, but did enhance recognition memory for positive pictures compared with loss anticipation. Second, there were large effects of feedback outcome on both recognition and recall of the pictures. Third, feedback outcome was not only associated with memory enhancement of the picture seen just before on the same trial, but also with enhanced recognition of the pictures on the next trial or two. For all of these effects, older adults showed similar patterns of results and there were no significant age interactions. Our sample size provided good power to detect large effects ( $96 \%$ according to Cohen, 1988), therefore our results suggest there are not large age differences in how reward anticipation and outcome modulate memory. While previous research shows that older adults are impaired at learning associations between cues and rewards (Mell et al., 2005, 2009; Eppinger et al., 2010; Eppinger and Kray, 2011), our findings are consistent with previous studies that found that manipulating the reward value of outcomes modulated memory for other information similarly in younger and older adults (Weiler et al., 2008; Eppinger et al., 2010).

\section{HOW CAN THE CURRENT FINDINGS BE RECONCILED WITH AGE-RELATED DECLINES IN DOPAMINE?}

These results are especially intriguing when considered against the backdrop of age-related declines in dopamine-related systems in the brain. Consistent with the idea that receiving rewards may signal the presence of worthwhile information for learning, there is growing evidence that midbrain dopamine regions that respond to motivationally relevant information interact with the hippocampus to enhance memory for that information (Shohamy and Adcock, 2010). Research with animals reveals that dopamine facilitates encoding novel information and increases the persistence of memory for that information (Jay, 2003; O'Carroll et al., 2006; Rossato et al., 2009; Bethus et al., 2010). In the rat hippocampus, blocking dopamine (D1/D5) receptors prevented exposure to a novel environment from facilitating long-term potentiation (Li et al., 2003). Furthermore, in healthy people as well as in Alzheimer's disease patients, D2 receptor binding in the hippocampus is positively correlated with memory function (Kemppainen et al., 2003; Takahashi et al., 2008; MacDonald et al., 2009).

There is abundant research demonstrating age-related declines in various aspects of dopaminergic systems in the brain (Bäckman et al., 2006; Backman et al., 2010). These declines are seen in particular in the striatal regions (Suhara et al., 1991; Wang et al., 1998), for instance, striatal dopamine levels decrease by more than threefold from those in their 30s to those in their late 80 s (Haycock et al., 2003).
However, the behavioral consequences of age-related declines in dopamine systems are not yet well understood. One challenge is that dopamine synthesis is affected differentially by age in different brain regions. For instance, in a study in which uptake of L-DOPA decreased $4.2 \%$ per decade in the putamen and $5.4 \%$ in the caudate nucleus, no age-related differences were found in the midbrain (Ota et al., 2006). However, larger declines were found in prefrontal, parietal, and medial temporal cortices, with as high as $16.4 \%$ decline per decade seen in the dorsolateral prefrontal cortex (Ota et al., 2006). D2 and D3 receptor subtypes also decline at different rates in different brain regions (Kaasinen et al., 2000, 2002). In addition to these regional differences in age-related decline in dopamine, there are also differences in how the various components of the dopaminergic brain systems are affected by age. For instance, a study that compared levels of six different presynaptic dopaminergic markers in postmortem striatum in people aged 1 day to 103 years old found that striatal dopamine levels decreased during adult aging but that the proteins involved in its biosynthesis and compartmentation were relatively preserved (Haycock et al., 2003).

While our study provides no direct evidence about dopaminergic processing, one candidate mechanism for the reward-related memory modulations shown by our participants are fluctuations in dopamine elicited by reward anticipation and outcomes that modulate hippocampal encoding. With this mechanism, for older adults to show effective reward-related modulation of memory encoding, they would need to have: (A) modulations in phasic dopamine activity during reward anticipation or outcomes and (B) maintained effectiveness of dopamine to modulate new memory encoding.

There is little direct evidence yet in the literature to indicate whether older adults do or do not have these necessary preconditions. However, some functional neuroimaging studies have examined brain activity in the striatum, a target region for midbrain dopaminergic neurons (Lyndbalta and Haber, 1994). Such studies have found that older adults show robust responses in ventral and/or dorsal striatum to positive outcomes (Schott et al., 2007; Cox et al., 2008; Mell et al., 2009; Samanez-Larkin et al., 2010). Results from studies examining reward anticipation are more mixed, with older adults showing less ventral striatal activation than younger adults in some cases (Schott et al., 2007; Dreher et al., 2008) but not in all studies (Samanez-Larkin et al., 2007). There are also age differences in whether stronger responses to outcomes are seen early or late in the learning phase of a probabilistic learning task (Mell et al., 2009). Thus, although there may be age differences in the conditions or types of stimuli that evoke striatal activation, evidence suggests there is some degree of intact functional signaling of reward in dopaminergic pathways among older adults.

In terms of the second precondition - dopamine modulated memory encoding - a recent study found that older participants showed reliable memory impairments/enhancements from a D2 antagonist/agonist manipulation whereas younger adults did not show significant memory modulation from the D2 manipulation (Morcom et al., 2010). In addition, administration of levadopa improved older adults' encoding of a motor memory more than it did for younger adults (who were already at a high level; Floel et al., 
2005). Thus, there is evidence that dopaminergic agents modulate memory-encoding processes for older adults.

In summary, previous evidence indicates that older adults show robust responses to reward outcomes and also show robust dopaminergic modulation of memory encoding. These findings suggesting intact aspects of reward processing and dopamine function may help explain our behavioral findings that reward processing can modulate incidental memory encoding as effectively for older adults as for younger adults.

\section{FUTURE DIRECTIONS}

Future research should explore the neural mechanisms underlying these effects, as well as further delineating the nature of the behavioral effects. For instance, are the effects driven more by positive outcomes enhancing memory or by negative outcomes impairing memory? Do outcomes modulate memory more or less for representations that are initially weak? In our study, encoding was incidental; would an intentional memory encoding task also yield significant modulation by outcomes? Addressing

\section{REFERENCES}

Adcock, R. A., Thangavel, A., WhitfieldGabrieli, S., Knutson, B., and Gabrieli, J. D. E. (2006). Rewardmotivated learning: mesolimbic activation precedes memory formation. Neuron 50, 507-517.

Anderson, J. R., and Schooler, L. J. (2000). "The adaptive nature of memory," in Oxford Handbook of Memory, eds E. Tulving and F. I. M. Craik (London: Oxford University Press), 557-570.

Backman, L., Lindenberger, U., Li, S. C., and Nyberg, L. (2010). Linking cognitive aging to alterations in dopamine neurotransmitter functioning: recent data and future avenues. Neurosci. Biobehav. Rev. 34, 670-677.

Bäckman, L., Nyberg, L., Linderiberger, U., Li, S. C., and Farde, L. (2006). The correlative triad among aging, dopamine, and cognition: current status and future prospects. $\mathrm{Neu}$ rosci. Biobehav. Rev. 30, 791-807.

Bethus, I., Tse, D., and Morris, R. G. M. (2010). Dopamine and memory: modulation of the persistence of memory for novel hippocampal NMDA receptor-dependent paired associates. J. Neurosci. 30, 1610-1618.

Bialleck, K. A., Schaal, H. P., Kranz, T. A., Fell, J., Elger, C. E., and Axmacher, N. (2011). Ventromedial prefrontal cortex activation is associated with memory formation for predictable rewards. PLoS ONE 6, el6695. doi:10.1371/journal.pone.0016695

Brown, J. I., Fishco, V. V., and Hanna, G. (1993). Nelson-Denny Reading Test: Manual for Scoring and Interpretation. Itasca, IL: Riverside.
Callan, D. E., and Schweighofer, N. (2008). Positive and negative modulation of word learning by reward anticipation. Hum. Brain Mapp. 29, 237-249.

Castel, A. D. (2008). The adaptive and strategic use of memory by older adults: evaluative processing and value-directed remembering. Psychol. Learn. Motiv. 48, 225-270.

Castel, A. D., Benjamin, A. S., Craik, F. I. M., and Watkins, M. J. (2002). The effects of aging on selectivity and control in short-term recall. Mem. Cognit. 30, 1078-1085.

Cohen, J. (1988). Statistical Power Analysis for the Behavioral Sciences. Hillsdale, NJ: Lawrence Erlbaum.

Cox, K. M., Aizenstein, H. J., and Fiez, J. A. (2008). Striatal outcome processing in healthy aging. Cogn. Affect.

Dreher, J. C., Meyer-Lindenberg, A., Kohn, P., and Berman, K. F. (2008). Age-related changes in midbrain dopaminergic regulation of the human reward system. Proc. Natl. Acad. Sci. U.S.A. 105, 15106-15111.

Eppinger, B., Herbert, M., and Kray, J. (2010). We remember the good ing and memory. Neurobiol. Learn. Mem. 93, 515-521.

Eppinger, B., and Kray, J. (2011). To choose or to avoid: age differences in learning from positive and negative feedback. J. Cogn. Neurosci. 23, 41-52.

Floel, A., Breitenstein, C., Hummel, F., Celnik, P., Gingert, C., Sawaki, L., Knecht, S., and Cohen, L. G. (2005). Dopaminergic influences on formation of a motor memory. Ann. Neurol. 58, 121-130. Behav. Neurosci. 8, 304-317. things: age differences in learn-

such issues would further clarify the specific mechanisms of this outcome-modulated memory effect.

\section{CONCLUSION}

This study revealed strong associations between outcomes on each trial and incidental memory for the target on that trial, as well as for incidental memory for the next target. This relationship between outcomes and memory was not dependent on response time performance and so suggests that receiving a good outcome creates a brief window of enhanced memory encoding. This association between good outcomes and memory was as strong for older adults as it was for younger adults, suggesting that the ability of reward processing to modulate memory remains robust in older age.

\section{ACKNOWLEDGMENTS}

Mara Mather and Andrej Schoeke, Davis School of Gerontology, University of Southern California. This research was supported by NIA grants R01AG038043 and K02AG032309.

Haycock, J. W., Becker, L., Ang, L., Furukawa, Y., Hornykiewicz, O., and Kish, S. J. (2003). Marked disparity between age-related changes in dopamine and other presynaptic dopaminergic markers in human striatum. J. Neurochem. 87, 574-585.

Hedden, T., and Gabrieli, J. D. E. (2004). Insights into the ageing mind: a view from cognitive neuroscience. Nat. Rev. Neurosci. 5, 87-96.

Jay, T. M. (2003). Dopamine: a potential substrate for synaptic plasticity and memory mechanisms. Prog. Neurobiol. 69, 375-390.

Kaasinen, V., Kemppainen, N., Nagren, K., Helenius, H., Kurki, T., and Rinne, J. O. (2002). Age-related loss of extrastriatal dopamine D2-like receptors in women. J. Neurochem. 81, 1005-1010.

Kaasinen, V., Vilkman, H., Hietala, J., Nagren, K., Helenius, H., Olsson, H., Farde, L., and Rinne, J. (2000). Agerelated dopamine D2/D3 receptor loss in extrastriatal regions of the human brain. Neurobiol. Aging 21, 683-688.

Kemppainen, N., Laine, M., Laakso, M. P., Kaasinen, V., Nagren, K., Vahlberg, T., Kurki, T., and Rinne, J. O. (2003). Hippocampal dopamine D2 receptors correlate with memory functions in Alzheimer's disease. Eur. J. Neurosci. 18, 149-154.

Kensinger, E. A., Garoff-Eaton, R. J., and Schacter, D. L. (2007). Effects of emotion on memory specificity in young and older adults. J. Gerontol. B Psychol. Sci. Soc. Sci. 62, 208-215.

Knutson, B., Adams, C. M., Fong, G. W., and Hommer, D. (2001).
Anticipation of increasing monetary reward selectively recruits nucleus accumbens. J. Neurosci. 21, RC159.

Kornell, N., Castel, A. D., Eich, T. S., and Bjork, R. A. (2010). Spacing as the friend of both memory and induction in young and older adults. Psychol. Aging 25, 498-503.

Li, S. M., Cullen, W. K., Anwyl, R., and Rowan, M. J. (2003). Dopaminedependent facilitation of LTP induction in hippocampal CA1 by exposure to spatial novelty. Nat. Neurosci. 6, 526-531.

Luo, L., and Craik, F. I. M. (2008). Aging and memory: a cognitive approach. Can. J. Psychiatry 53, 346-353.

Lyndbalta, E., and Haber, S. N. (1994). The organization of midbrain projections to the striatum in the primate: sensorimotor-related striatum versus ventral striatum. Neuroscience 59, 625-640.

MacDonald, S. W. S., Cervenka, S., Farde, L., Nyberg, L., and Backman, L. (2009). Extrastriatal dopamine D2 receptor binding modulates intraindividual variability in episodic recognition and executive functioning. Neuropsychologia 47, 2299-2304.

Macmillan, N. A., and Creelman, C. D. (1991). Detection Theory: A User's Guide. New York: Cambridge University Press.

Marschner, A., Mell, T., Wartenburger, I., Villringer, A., Reischies, F. M. and Heekeren, H. R. (2005). Rewardbased decision-making and aging. Brain Res. Bull. 67, 382-390.

Mather, M. (2010). Aging and cognition. Wiley Interdiscip. Rev. Cogn. Sci. 1, 346-362. 
Mather, M., and Sutherland, M. R. (2011). Arousal-biased competition in perception and memory. Perspect. Psychol. Sci. 6, 114-133.

Mell, T., Heekeren, H. R., Marschner, A., Wartenburger, I., Villringer, A., and Reischies, F. M. (2005). Effect of aging on stimulus-reward association learning. Neuropsychologia 43, 554-563.

Mell, T., Wartenburger, I., Marschner, A., Villringer, A., Reischies, F. M., and Heekeren, H. R. (2009). Altered function of ventral striatum during reward-based decision making in old age. Front. Hum. Neurosci. 3:34. doi:10.3389/neuro.09.034.2009

Mitchell, K. J., Johnson, M. K., Raye, C. L., Mather, M., and D'Esposito, M. (2000). Aging and reflective processes of working memory: binding and test load deficits. Psychol. Aging 15, 527-541.

Morcom, A. M., Bullmore, E. T., Huppert, F. A., Lennox, B., Praseedom, A., Linnington, H., and Fletcher, P. C. (2010). Memory encoding and dopamine in the aging brain: a psychopharmacological neuroimaging study. Cereb. Cortex 3, 743-757.

Naveh-Benjamin, M., Guez, J., Kilb, A., and Reedy, S. (2004). The associative memory deficit of older adults: further support using face-name associations. Psychol. Aging 19, 541-546.

O'Carroll, C. M., Martin, S. J., Sandin, J., Frenguelli, B., and Morris, R. G. M. (2006). Dopaminergic modulation of the persistence of one-trial hippocampus-dependent memory. Learn. Mem. 13, 760-769.

Ota, M., Yasuno, F., Ito, H., Seki, C., Nozaki, S., Asada, T., and Suhara, T. (2006). Age-related decline of dopamine synthesis in the living human brain measured by positron emission tomography with L-[betaC-11]DOPA. Life Sci. 79, 730-736.

Radloff, L. S. (1977). The CES-D Scale: a self-report depression scale for research in the general population. Appl. Psychol. Meas. 1, 385-401.

Rossato, J. I., Bevilaqua, L. R. M., Izquierdo, I., Medina, J. H., and Cammarota, M. (2009). Dopamine controls persistence of long-term memory storage. Science 325, 1017-1020.

Samanez-Larkin, G. R., Gibbs, S. E. B., Khanna, K., Nielsen, L., Carstensen, L. L., and Knutson, B. (2007). Anticipation of monetary gain but not loss in healthy older adults. Nat. Neurosci. 10, 787-791.

Samanez-Larkin, G. R., Kuhnen, C. M., Yoo, D. J., and Knutson, B. (2010). Variability in nucleus accumbens activity mediates age-related suboptimal financial risk taking. J. Neurosci. 30, 1426-1434.

Schott, B. H., Niehaus, L., Wittmann, B. C., Schutze, H., Seidenbecher, C. I., Heinze, H. J., and Düzel, E. (2007). Ageing and early-stage Parkinson's disease affect separable neural mechanisms of mesolimbic reward processing. Brain 130, 2412-2424.

Shohamy, D., and Adcock, R. A. (2010). Dopamine and adaptive memory.
Trends Cogn. Sci. (Regul. Ed.) 14 464-472.

Suhara, T., Fukuda, H., Inoue, O. Itoh, T., Suzuki, K., Yamasaki, T., and Tateno, Y. (1991). Age-related changes in human D1-dopamine receptors measured by positron emission tomography. Psychopharmacology (Berl.) 103, 41-45.

Takahashi, H., Kato, M., Takano, H. Arakawa, R., Okumura, M., Otsuka, T., Kodaka, F., Hayashi, M., Okubo, Y., Ito, H., and Suhara, T. (2008). Differential contributions of prefrontal and hippocampal dopamine D-1 and D-2 receptors in human cognitive functions. J. Neurosci. 28, 12032-12038.

Wang, Y., Chan, G. L. Y., Holden, J. E., Dobko, T., Mak, E., Schulzer, M., Huser, J. M., Snow, B. J., Ruth, T. J., Calne, D. B., and Stoessl, A. J. (1998). Age-dependent decline of dopamine D1 receptors in human brain: a PET study. Synapse 30, 56-61.

Watson, D., Clark, L. A., and Tellegen, A. (1988). Development and validation of brief measures of positive and negative affect: the PANAS scales. $J$. Pers. Soc. Psychol. 54, 1063-1070.

Weiler, J. A., Bellebaum, C., and Daum, I. (2008). Aging affects acquisition and reversal of reward-based associative learning. Learn. Mem. 15, 190-197.

Wittmann, B. C., Daw, N. D., Seymour, B., and Dolan, R. J. (2008a). Striatal activity underlies noveltybased choice in humans. Neuron 58, 967-973.

Wittmann, B. C., Schiltz, K., Boehler, C. N., and Duzel, E. (2008b).
Mesolimbic interaction of emotional valence and reward improves memory formation. Neuropsychologia $46,1000-1008$.

Wittmann, B. C., Schott, B. H., Guderian, S., Frey, J. U., Heinze, H. J., and Duzel, E. (2005) Reward-related fMRI activation of dopaminergic midbrain is associated with enhanced hippocampus-dependent long-term memory formation. Neuron 45, 459-467.

Conflict of Interest Statement: The authors declare that the research was conducted in the absence of any commercial or financial relationships that could be construed as a potential conflict of interest.

Received: 11 September 2011; paper pending published: 21 September 2011; accepted: 01 November 2011; published online: 21 November 2011.

Citation: Mather $M$ and Schoeke A (2011) Positive outcomes enhance incidental learning for both younger and older adults. Front. Neurosci. 5:129. doi: 10.3389/fnins.2011.00129

This article was submitted to Frontiers in Decision Neuroscience, a specialty of Frontiers in Neuroscience.

Copyright (c) 2011 Mather and Schoeke. This is an open-access article subject to a non-exclusive license between the authors and Frontiers Media SA, which permits use, distribution and reproduction in other forums, provided the original authors and source are credited and other Frontiers conditions are complied with. 